\title{
Utilisation et mise en forme des récits de maladie dans l'expérimentation de l'électricité médicale
}

\author{
François Zanetti \\ DANS DiX-HUITIÈME SIÈCLE 2015/1 (N²4), PAGES 181 À 196 \\ Éditions Société FrançAise d'ÉTude du DiX-Huitième Siècle
}

ISSN $0070-6760$

ISBN 9782707186317

DOI 10.3917/dhs.047.0181

Article disponible en ligne à l'adresse

https://www.cairn.info/revue-dix-huitieme-siecle-2015-1-page-181.htm

\author{
CAIRN I \\ MATIÈRES À RÉFLEXION
}

Découvrir le sommaire de ce numéro, suivre la revue par email, s'abonner...

Flashez ce QR Code pour accéder à la page de ce numéro sur Cairn.info.

Distribution électronique Cairn.info pour Société Française d'Étude du Dix-Huitième Siècle.

La reproduction ou représentation de cet article, notamment par photocopie, n'est autorisée que dans les limites des conditions générales d'utilisation du site ou, le cas échéant, des conditions générales de la licence souscrite par votre établissement. Toute autre reproduction ou représentation, en tout ou partie, sous quelque forme et de quelque manière que ce soit, est interdite sauf accord préalable et écrit de l'éditeur, en dehors des cas prévus par la législation en vigueur en France. Il est précisé que son stockage dans une base de données est également interdit. 


\section{UTILISATION ET MISE EN FORME DES RÉCITS DE MALADIE DANS L'EXPÉRIMENTATION DE L'ÉLECTRICITÉ MÉDICALE}

Une des premières initiatives de la Société royale de médecine après sa création en 1776 est de confier à Pierre Mauduyt de la Varenne l'évaluation des propriétés thérapeutiques de l'électricité. Cette expérience répond à l'engouement contemporain pour les fluides invisibles et le développement de traitements électriques à Paris et dans les provinces. La Société y met en œuvre ses prérogatives dans l'étude des maladies épidémiques et de l'influence de l'atmosphère sur la santé ainsi que sa responsabilité dans la surveillance des remèdes ${ }^{1}$.

Pour mener à bien cette expérience particulière, Mauduyt de la Varenne propose chez lui un traitement pour les personnes souffrant de maladies chroniques, au premier rang desquelles figure la paralysie. Durant deux années (1777-1779), quatre-vingt-deux malades y sont électrisés. C'est à partir des archives et du compte rendu publié de ces séances que nous voudrions interroger les enjeux de la mise en forme des récits de maladie dans le contexte de l'expérimentation d'une nouvelle méthode de traitement ${ }^{2}$.

1. Paul Delaunay, Le Monde médical parisien au $18^{e}$ siècle, Paris, Jules Rousset, 1906, p. 308-330; Charles Coulston Gillispie, Science and Polity in France. The End of the Old Regime, Princeton, Princeton University Press, 1980, p. 28-33 et 194-203; Caroline Hannaway, Medicine, Public Welfare and the State in Eighteenth-Century France. The Société royale de médecine de Paris (1776-1793), thèse de doctorat, Johns Hopkins University, Baltimore, 1974 (dactyl.).

2. Académie nationale de médecine, Paris, Fonds de la Société royale de médecine (désormais SRM), 118, dossiers 1 à 82. Le compte rendu du traitement a été publié en 1780 dans le premier volume de l'histoire de la Société royale de médecine : Pierre Mauduyt de la Varenne, "Mémoire sur le traitement électrique administré à quatre-vingt-deux malades ", Histoire de la Société royale de méde- 
Le poids de l'idiosyncrasie dans les conceptions médicales justifie l'importance, pour évaluer l'efficacité d'un nouveau remède, de faire raconter par le malade les épisodes de la maladie et de rendre compte des événements qui la ponctuent ${ }^{3}$. Cette nécessité entre en tension avec le nombre de malades et le recours à des " technologies de papier " sophistiquées qui visent à dépersonnaliser les faits ${ }^{4}$. Ces récits mettent en valeur d'autres tensions dans les conceptions de la maladie, caractéristiques des débats nosologiques du second $18^{\mathrm{e}}$ siècle $^{5}$ : comment identifier une maladie? Le récit permet d'articuler les causes et les symptômes pour la définir, tant du point de vue du médecin, que du malade et de son entourage $e^{6}$. Une troisième tension apparaît lors de la publication. La maladie qui a été racontée à plusieurs voix dans le cadre de la rencontre thérapeutique vise désormais un lectorat plus large. La nécessaire publicité du traitement doit alors ménager la confiance des malades qui se sont livrés dans l'espoir de guérir ${ }^{7}$.

cine..., Avec les Mémoires de médecine et de physique médicale... tirés des registres de cette société, Années 1777 et 1778, Paris, Didot le jeune, 1780, p. 199-431.

3. Séverine Pilloud, Les Mots du corps. Expérience de la maladie dans les lettres de patients à un médecin du $18^{e}$ siècle : Samuel Auguste Tissot, Lausanne, BHMS, 2013, p. 59-61.

4. Volker Hess et J. Andrew Mendelsohn, "Case and Series : Medical Knowledge and Paper Technology, 1600-1900", History of Science, 48, 2010, p. 287-314. Les controverses contemporaines autour de l'inoculation révèlent également cette tension entre rhétorique du nombre et histoires individuelles, voir Catriona Seth, "L'inoculation de la variole : un révélateur des liens sociaux ", Dix-huitième siècle, 41, 2009, p. 147.

5. Jean-Pierre Peter, «Les mots et les objets de la maladie : remarques sur les épidémies et la médecine dans la société française de la fin du $18^{\mathrm{e}}$ siècle ", Revue historique, 246, 1971, p. 13-38.

6. Micheline Louis-Courvoisier et Séverine Pilloud, « Le malade et son entourage au $18^{\mathrm{e}}$ siècle : les médiations dans les consultations épistolaires adressées au Dr Tissot ", Revue médicale de suisse romande, 120, 2000, p. 939-944.

7. Micheline Louis-Courvoisier et Philip Rieder, "Introduction : genèse d'une éthique médicale ", dans Les Honoraires médicaux et autres mémoires d'éthique médicale, Paris, Classiques Garnier, coll. "Bibliothèque du $18^{\mathrm{e}}$ siècle ", 2011, p. 36-42. 


\section{Le mémoire : des récits au tableau}

Le " mémoire sur le traitement électrique administré à quatrevingt-deux malades " est un document hétérogène qui témoigne de la recomposition des procédures d'évaluation de l'efficacité thérapeutique. Au cours du $18^{\mathrm{e}}$ siècle, la place, la fonction et la forme des récits de cas dans l'administration de la preuve scientifique et médicale sont redéfinis ${ }^{8}$. Sur le double modèle des sciences physiques et de l'arithmétique politique, la quantification prend une part croissante dans la rhétorique de l'expérience. L'organisation générale du mémoire traduit une volonté d'épuration de la singularité des observations pour construire des faits incontestables, par le recours au résumé, à la numérotation et au tableau. Néanmoins, les récits de la maladie et du traitement suivi pour chaque malade occupent 210 des 236 pages du mémoire (p. 201-411). Mauduyt de la Varenne commence son mémoire par une explicitation de la procédure qu'il a mise en œuvre et qui rend compte de la structure du document : "Quand j'ai pensé, avec ceux de mes confrères qui avaient été appelés, qu'un malade était dans le cas d'être électrisé, j'ai rédigé un précis historique de la maladie que j'allais entreprendre de combattre; j'ai tâché, autant qu'il m’a été possible, de rédiger ce précis conjointement avec le médecin ou le chirurgien qui avaient traité le malade dès l'origine de sa maladie pendant son cours. Je me suis réglé sur le récit du malade et sur celui de ses parents ou amis qui l'accompagnaient, quand je n'ai pu faire autrement. J'ai décrit ensuite son état actuel; et après avoir lu lentement aux assistants ce que je venais d'écrire en leur présence, je le leur ai fait signer ${ }^{9}$."

Le récit de la maladie est un élément primordial dans la procédure d'expérimentation. Mauduyt tient ensuite un journal où il transcrit les évolutions de l'état du malade au fur et à mesure de l'administration de l'électricité. Il décrit le dispositif matériel

8. Christian Licoppe, La Formation de la pratique scientifique. Le discours de l'expérience en France et en Angleterre (1630-1820), Paris, La Découverte, p. 243269; François Doublet, Expérience et ExpérIence particulière, Encyclopédie méthodique. Médecine, t. 6, 1793, p. 173-187.

9. Pierre Mauduyt de la Varenne, "Mémoire sur le traitement électrique... ", art. cité, p. 199-200. 
qui a permis de consigner les observations : "Chaque journal, portant une étiquette sur laquelle est écrit le nom de celui qu'il concerne, est resté, pendant tout le temps du traitement, exposé sur une table dans la pièce où je reçois les malades. Cette pièce a été constamment ouverte pendant les heures du traitement aux médecins, aux chirurgiens et aux physiciens qui ont désiré d'y entrer [...]. Chacun a pu, comme il l'a jugé à propos, prendre les journaux, les lire, interroger les malades, examiner leur état devant moi ou dans mon absence. En commençant la journée, j'interrogeais les malades, et j'écrivais, d'après leurs réponses, les articles qui sont consignés sur les journaux; je priais ensuite ceux de mes confrères qui étaient présents de signer les articles que je venais de rédiger. Avant que personne signât, ni que je regardasse aucun article comme arrêté, je le lisais tout haut à celui qu'il concernait, en présence des autres malades devant lesquels je l'avais interrogé " (p. 200). Ces cahiers ont fait l'objet durant le traitement de pratiques de lecture ou d'écriture diverses, individuelles ou collectives, par les malades ou les praticiens. Ils ont été classés conformément à l'organisation définitive du volume publié : "Je divise les malades dont je parle suivant les maux dont ils étaient attaqués; je les présente d'après la durée de leur traitement, et selon le soulagement qu'ils ont obtenu " (p. 201). Les maladies dont il s'agit sont les "paralysies ", les "stupeurs et engourdissements ", les "rhumatismes ", les "rhumatismes goutteux ", le "lait épanché ", les "surdités ", les "maladies des yeux " et la "suppression et défaut de règles ». La paralysie concerne 51 des 82 malades traités.

À l'intérieur de cette organisation nosologique, les malades sont classés en fonction de l'avis du médecin sur la durée du traitement (suffisante ou insuffisante) et de son succès (guérison, améliorations, aucun). Enfin, à chaque malade est attribué un numéro, " afin de pouvoir en reparler dans la suite, et de rapporter à leur égard les faits qui mériteront d'être cités ${ }^{10}$ ".

Dans le "tableau des malades qui ont été électrisés " (inséré à la p. 410 et reproduit ici p. 196), une ligne est consacrée à chaque malade, dans l'ordre du mémoire. Le numéro du malade figure

10. Pierre Mauduyt de la Varenne, "Mémoire sur le traitement... ", art. cité, p. 201. 
dans la première colonne. Viennent ensuite le numéro de la page, le nom du malade, son âge, la durée de la maladie et ses causes probables. Les trois dernières colonnes, qui représentent plus de la moitié de la largeur du tableau, concernent le traitement et ses conséquences. Le tableau donne ainsi une vision synthétique du mémoire. La maladie y est résumée à sa durée et à sa cause, réduite à quelques mots au plus. Il informe également sur la manière dont l'auteur entendait que son mémoire soit manipulé et lu : il représente une table pour circuler dans le mémoire et permet aussi la comparaison des cas selon les intitulés de chaque colonne.

Le " résumé " (p. 411-426) est un second instrument pour orienter le lecteur dans son usage du livre et des histoires. Il représente un intermédiaire entre les cas et le tableau. Plus exactement, il consiste en une proposition de lecture du tableau qui renvoie aux journaux des malades. Ainsi, pour la première catégorie, celle des paralytiques qui ont suivi le traitement aussi longtemps que Mauduyt leur a conseillé et qui en ont été soulagés (malades 1 à 10 ) on peut lire : « $3^{\circ}$. Il est possible que la paralysie reconnaissait pour causes le relâchement des fibres et une congestion humorale dans six des dix malades soulagés. (Voy. les ${ }^{\text {os }}$ I, II, III, V, VIII, X.) Elle était la suite d'une frayeur violente et subite dans le malade $\mathrm{n}^{\circ} \mathrm{IV}$; elle paraissait être celle de la pléthore sanguine dans la malade $\mathrm{n}^{\circ} \mathrm{VI}$; celle d'une humeur portée d'abord à la peau et ensuite répercutée, dans le malade $\mathrm{n}^{\circ} \mathrm{IX}$ : rien n'éclaire sur la cause de la paralysie du malade $n^{\circ}$ VII » (p. 411-412). Il s'agit en fait de la lecture verticale de la sixième colonne (" causes probables de la maladie ») pour les dix premières lignes du tableau ${ }^{11}$.

\section{La lecture comme expérience}

Les modalités mouvantes de l'administration de la preuve aboutissent à un document composite et à une stratégie argumentative complexe qui s'incarne dans les trois formes complémentaires que prennent les observations dans le mémoire (journaux, résumé, tableau). On est frappé par l'utilisation limitée que Mauduyt

11. Volker Hess et J. Andrew Mendelsohn, "Case and Series... ", art. cité, p. 292. À propos des observations du médecin viennois De Haen, les auteurs font l'hypothèse de la mise en texte de tableaux d'observations cliniques. 
fait de l'élaboration tabulaire de ses observations. Même dans le résumé, le modèle narratif prend souvent le pas sur la lecture verticale et l'appropriation de la " technologie de papier " n'aboutit pas à l'évaluation quantitative des résultats. Le tableau et le résumé renvoient essentiellement au récit des journaux. Mauduyt s'en explique en partie : "Ce serait le lieu de conclure en cet endroit, relativement à l'utilité ou l'inutilité de l'électricité en général, en particulier relativement à l'usage qu'on en peut faire dans la paralysie, et d'examiner quelles sont les espèces de cette maladie auxquelles elle peut le mieux convenir. Mais je m'abstiendrai d'exposer mon sentiment sur ces objets, dont je remets et dont j'abandonne absolument la décision aux médecins, à qui seuls elle appartient, et qui prononceront; soit d'après les faits que j'ai rapportés, soit d'après ceux qui ont été annoncés dans les différents écrits rendus publics» (p. 426). Plus loin, il ajoute : " Je crois que pour prononcer sur une chose, il faut la connaitre dans toutes ses parties, sous tous ses aspects. Mon propre sentiment, dont je ne parle pas, doit, pour être raisonnable, émaner des faits dont j'ai été témoin; celui des lecteurs doit avoir la même base : je leur devais donc compte des faits, et je devais les en rendre témoins, comme je l'ai été, autant que la chose est possible par le récit» (p. 430-431). Pour Mauduyt, le récit et la pluralité de voix visent à l'exhaustivité et sont un moyen nécessaire à l'établissement de faits. Le mode de présentation des cas doit faire du lecteur un témoin actif. La lecture est alors construite comme une forme de réplication de l'expérience. L'exploitation limitée des ressources des « technologies de papier " utilisées est aussi caractéristique des dernières décennies du $18^{\mathrm{e}}$ siècle et tient notamment à la permanence du modèle idiosyncrasique et narratif de la maladie ${ }^{12}$. Malgré la volonté qu'il en exprime Mauduyt ne parvient pas véritablement à établir « les espèces de $[. .$.$] maladie » pour lesquelles l'électricité peut être utile$

12. Jean-Baptiste Fressoz, L'Apocalypse joyeuse. Une histoire du risque technologique, Paris, Le Seuil, 2012, p. 68; Catriona Seth, "L'inoculation de la variole : un révélateur des liens sociaux ", art. cité, p. 147; Volker Hess et J. Andrew Mendelsohn, "Case and Series... ", art. cité, p. 295; Michel Foucault, Sécurité, territoire, population. Cours au Collège de France. 1977-1978, Paris, Gallimard, Le Seuil, p. 61-62. 
et renvoie à des histoires individuelles de maladie et de traitement pour en évaluer l'efficacité.

\section{Identifier la maladie}

Pour le médecin, décrire la maladie nécessite de recueillir le récit du malade pour connaître les événements qui la constituent. Il lui faut ensuite donner forme et sens à ces maux selon une logique nosographique pour établir dans quels cas l'électricité est le remède indiqué.

Parfois, Mauduyt rencontre des difficultés pour recueillir le récit. Dans le cas de Bodin, les tensions entre la nouvelle Société royale de médecine et la Faculté de médecine de Paris perturbent la transmission entre Le Clerc, médecin ordinaire du malade et Mauduyt $^{13}$. Il a alors recours à l'entourage du malade pour connaître son histoire. Le dialogue peut également être empêché entre le médecin et le malade. Il peut s'agir de questions linguistiques, comme dans le cas d'un coiffeur allemand : "Je n'ai pu savoir du malade qui a beaucoup de difficultés à parler français, comment sa paralysie lui a pris : elle est venue à la suite d'une chute de cheval et après avoir été exposé à de grands froids ${ }^{14}$. " Il peut aussi s'agir de difficultés physiques à s'exprimer quand la paralysie affecte la bouche ou les cordes vocales. Lorsque l'huissier Dagneau consulte Mauduyt pour mutisme, c'est sa femme qui prend la parole pour présenter la situation ${ }^{15}$. Mauduyt note aussi la faiblesse des compétences ou du désir à parler de soi dans le cas de Castres, premier Suisse de l'abbaye Saint-Martin-des-Champs ${ }^{16}$ : «Le malade avait toute la bonhomie propre à sa nation, mais il n'était pas homme à m’instruire si ses urines déposaient, s'il transpirait plus qu'à l'ordi-

13. SRM 118, dossier 16. Ces éléments ne sont pas mentionnés dans le mémoire imprimé (p. 284-287).

14. SRM 118, dossier 20.

15. SRM 153, dossier 14.

16. Le suisse est un "domestique à qui l'on confie la garde d'une porte " (Dictionnaire de l'Académie française, $4^{\mathrm{e}}$ édition, Paris, Vve Brunet, 1762, p. 774). Dans les églises, les suisses sont chargés de prévenir les irrévérences et de veiller aux bonnes mœurs. 
naire. Je sais bien qu'il crachait souvent, mais j'ignore si la salivation était antérieure à l'électricité ${ }^{17}$."

Quel qu'en soit l'auteur, le récit est souvent fourni et doit permettre de définir la maladie dont souffre le malade pour établir pour quelles maladies l'électricité peut être utile. Mais, ce qui permet de définir et de classer une maladie n'est pas évident. Au-delà des catégories générales qui organisent le mémoire, Mauduyt peine à les identifier précisément. La nosologie est une préoccupation importante des médecins du $18^{\mathrm{e}}$ siècle $^{18}$. On peut opposer deux modèles d'identification et de classement : les symptômes et les causes. Dans l'article Pathologie de l'Encyclopédie, l'auteur écrit : "C'est une absurdité que de prétendre considérer et définir la maladie dépouillée de ses symptômes : cette abstraction métaphysique, absolument déplacée dans les sciences de faits, ne servirait qu'à obscurcir la connaissance des maladies en éloignant les phénomènes qui les caractérisent, et la rendrait incertaine en la pliant aux lois variables de théorie ${ }^{19}$. " En revanche, lorsqu'il s'agit de thérapeutique, l'auteur de l'article MaLADIE insiste sur la nécessité d'en connaître la cause : "La connaissance de la maladie dépend donc de la connaissance des actions, dont le vice est une maladie : il ne suffit pas d'en savoir le nom, il faut en connaître la cause prochaine : il est aisé de s'apercevoir qu'une personne est aveugle pour peu qu'on la considère; mais que s'ensuit-il de là pour sa guérison si elle est possible? il faut à cet égard, savoir ce qui l'a privé de la vue $[\ldots]^{20}$. " La perspective expérimentale et thérapeutique de Mauduyt justifie l'attention particulière portée à la cause que traduit notamment l'organisation du tableau synthétique.

17. Pierre Mauduyt de la Varenne, "Mémoire sur le traitement électrique... ", art. cité, p. 292.

18. Laurence Brockliss et Colin Jones, The Medical World of Early Modern France, Oxford, Clarendon Press, 1997, p. 434-436; Gilles Barroux, Philosophie, maladie et médecine au 18e siècle, Paris, H. Champion, 2008, p. 84-109; Roselyne Rey, Naissance et développement du vitalisme en France dans la deuxième moitié du $18^{e}$ siècle à la fin du Premier Empire, Oxford, Voltaire Foundation, 2000, p. 73.

19. Pathologie, Encyclopédie ou dictionnaire raisonné des sciences, des arts et des métiers, Paris, Le Breton, t. 12, 1765, p. 170.

20. Maladie, Encyclopédie, ouvr. cité, t. 9, p. 931. 


\section{Les caractères de la maladie}

Les cas relatés par Mauduyt mettent en évidence deux caractéristiques de la manière de concevoir les maladies au $18^{\mathrm{e}}$ siècle : elles se révèlent dans la durée et, pour les identifier, il est nécessaire de reprendre la biographie des malades. On remarque également la longévité du modèle humoral à travers la crainte de la pléthore et l'importance accordée aux émotions.

Dans les récits des malades et des médecins, l'établissement de la cause ouvre une chronologie particulière qui allie la brièveté de l'instant à la profondeur de l'existence ${ }^{21}$. Quand Monsieur Prévost écrit pour Mauduyt l'histoire de la paralysie qui affecte la partie gauche de son visage, il articule différentes temporalités : "Il y’a un mois que cette accident mest venue apres mette bien purgé pendant 10 a 12 jours sur les derniers jours je but beaucoup deau de citron bouillie avec du sucre/Il est a observer que les accides mont toujours incommodé/Cela ma pris dans la nuit sans avoir senty la moindre chose sy non le jour avant quelque malaisse dans la jambe. " Après avoir ainsi fait référence à la soudaineté de l'attaque et à son régime des dix jours la précédant, il ajoute : "je nait jamai eut aucune maladie daucune espece sinon une maladie de nerf Il y a six ans dont il m'en est resté une sensibilité/je bois mange dort bien et ne sent aucune douleur nulle part joublie de dire que j'ay mauvais estomacq Digerant difficilement/tous les ans j'ay des rhumes de cerveau qui me font couler des eaux rousse par les narine cest année je nen ais pas eût./je suis sujette a la pituite ${ }^{22}$ ". C'est le retour régulier des rhumes de cerveau qui intéresse Mauduyt ${ }^{23}$. Dans le mémoire, il écrit : "il n'avait point eu de rhume de cerveau l'année à la fin de laquelle il fut attaqué de paralysie aux muscles du visage. Il est donc probable que la suppression d'une excrétion séreuse qui coulait plusieurs fois tous les

21. Voir Séverine Pilloud, Les Mots du corps, ouvr. cité, p. 70-71 et p. 227-231.

22. SRM 118, dossier 2.

23. Sur l'importance des évacuations périodiques dans la définition de la santé à l'époque moderne, voir Gianna Pomata, "Menstruating Men : Similarity and Difference of the Sexes in Early Modern Medicine ", dans Generation and Degeneration, Tropes in Literature and History from Antiquity through Early Modern Europe, dir. Valeria Finucci et Kevin Brownlee, Durham, Duke University Press, 2001, p. 109-152. 
ans par les narines, qui n'avait pas eu lieu cette année, était la cause des accidents qui étaient survenus »(p. 206). Dans le tableau il résume : "congestion humorale".

La congestion, ou le défaut d'évacuation sont une étape récurrente des récits. On est frappé par la permanence du modèle humoral dans l'explication des maladies et de l'impératif évacuateur dans la thérapeutique qui informent l'adoption de l'électricité comme remède $^{24}$. Cela permet de rendre compte de l'attention portée aux règles et à leur suppression. Mauduyt leur consacre une catégorie (trois malades), mais la mention de la situation menstruelle des malades est systématique, quelles que soient les maladies dont elles sont affectées. Pour expliquer leur suppression chez une jeune malade restée anonyme, Mauduyt écrit, à la suite des médecins ordinaires : « $\mathrm{M}^{\mathrm{lle}}$ a connu une frayeur excessive qui a cependant diminué de quelque chose, quoiqu'elle soit encore très forte. La malade craint de se trouver seule le soir dans sa chambre ${ }^{25}$. " Dans le mémoire imprimé, on trouve : "Une demoiselle de quinze ans et demi, qui depuis deux ans avait atteint l'âge de la puberté, dont les évacuations périodiques étaient abondantes et suivaient leur cours ordinaire, entendit crier au feu à côté de chez elle au mois de janvier 1776; c'était au moment de l'évacuation; elle fut arrêtée subitement et de ce moment au trente décembre 1777 "(p. 408). L'association pathologique entre émotion et défaut d'évacuation est inversée dans le cas de Madame de la Trémolières qui a recours au traitement électrique pour soigner sa surdité : "il y a trois ans que Madame de La Trémoliere [...] a la suite d'un chagrin considérable dans lequel Mde retint ses larmes, fut subitement attaquée d'un mal tres considérable aux ieux ${ }^{26}$ ". La cause de la maladie est partagée entre le chagrin et le défaut d'évacuation des larmes.

24. Voir Philip Rieder, La Figure du patient au $18^{e}$ siècle, Genève, Droz, Bibliothèque des Lumières, 2010, p. 312-324 et Séverine Pilloud, Les Mots du corps, ouvr. cité, p. 182-186.

25. SRM 118, dossier 81.

26. SRM 118, dossier 74 . 


\section{Scansions : remèdes et progrès}

Parmi les étapes qui ponctuent le récit des maladies et, parfois, des guérisons, les thérapeutiques mobilisées et les capacités recouvrées apparaissent comme des jalons importants.

L'histoire de la maladie et celle des traitements sont dès lors souvent imbriquées, d'autant que l'électricité est utilisée pour soigner des maladies chroniques et comme un dernier recours dans un parcours à la fois symptomatique et thérapeutique. Les remèdes apparaissent même parfois comme une cause de la maladie, surtout lorsqu'ils ont été prescrits par un charlatan. C'est le cas dans la maladie de Madame Vanier, qui souffre de douleurs et d'un gonflement du genou : "six mois après, étant incommodée de corps au pied droit, elle y appliqua de la poix de bourgogne, on coupa les corps; les doigts s'enflerent et supurerent. l'hyver suivant Mde vanier outre la gêne dans le genouil gauche, en éprouvat aussi, mais a un degré plus léger, dans le col, les deux bras et la jambe droite. elle se mit alors entre les mains d'un charlatan qui lui fit prendre longtems des remedes sudorifiques et des purgatifs. il lui apliquat un topique sur les parties malades. ce topique excita des piquotements, un frémissement universel et la malade n'en put soutenir que la $1^{\text {re }}$ application. les membres se gonflerent, il y eut un oedême général ${ }^{27}$. " L'expérience de la maladie est caractérisée par la recherche et la participation à des traitements successifs dans l'espoir de guérir qui scandent les récits des maladies.

L'observation des progrès du malade traque le recul de la maladie. L'enjeu est de rendre ces évolutions sensibles pour le lecteur. Pour les paralytiques, on énumère les capacités retrouvées à agir : temps de parcours pour se rendre au traitement, aptitude à enlever ou remettre son chapeau, à s'alimenter de manière autonome, à manier une tabatière... Pour l'aveugle, les choses qu'il peut désormais voir. La focalisation fournit au lecteur un aperçu de l'environnement du traitement. C'est ainsi qu'est dévoilée la collection ornithologique de Mauduyt : Le matin du 20 juin 1778, Madame Dumont " distingue les objets plus nettement, placée sur l'isoloir en face d'une armoire remplie d'oiseaux, elle aperçoit les oiseaux et

27. SRM 118, dossier 48. 
un peu la couleur rouge d'un d'entre eux. " Après une amélioration, sa vue décline à nouveau : " elle a vu jusqu'aux étiquets des oiseaux placés devant elle quand elle est sur l'isoloir, elle ne les voit pas ce matin $^{28}$ ". Pour rendre sensible la maladie et ses évolutions, Mauduyt utilise d'autres techniques. Ainsi, l'écriture du malade fournitelle un moyen de mesurer l'aisance dans le mouvement des doigts. Madame Boitel écrit de sa main un petit billet conservé dans son dossier : " monsieur mauduit de varainne ma recommendé descrire/ je voudroy que ma main puise tracer nettement les sentiments de reconoisance que jay de tout ses bontés/je luy en fait mil-remerciement/jay lhonneur destre sa tres humble servante/boitel ${ }^{29}$.»

\section{Publier les cas : confidences, secret et confiance}

Alors que les récits des maladies jouent un rôle important dans l'administration de la preuve de l'efficacité du remède électrique, leur publication modifie la configuration de la relation thérapeutique. L'intimité d'existences singulières est soumise au scrutin d'un public élargi. La manière de présenter les cas dans le mémoire prend ainsi en compte les exigences d'une relative confidentialité ${ }^{30}$.

Un tiers des cas du mémoire sont anonymes. Mauduyt s'en explique dans le cas d'un certain Blondel : "Ce malade et ceux qui, comme lui, ne seront pas nommés, m’ont demandé à ne pas l'être, ou ce que j'avais en à dire ne le permettait pas $^{31} »$ (p. 248). Seules les mentions de la sexualité et des maux vénériens dans l'histoire des malades impliquent nécessairement l'anonymat. Mauduyt a intérêt à nommer les privilégiés pour donner une plus grande autorité à son traitement. L'abbé Maudoux a ainsi conscience de revêtir une importance particulière pour la réputation du nouveau remède. Il l'écrit avec humour à l'abbé Bergier ${ }^{32}$ : "Je suis payé

28. SRM 118, dossier 77.

29. SRM 118, dossier 6.

30. Voir Louis Odier, Les Honoraires médicaux et autres mémoires d'éthique médicale, éd. cit., "Introduction ", p. 37-42.

31. SRM 118, dossier 9.

32. Voir Sylviane Albertan-Coppola, L'Abbé Nicolas-Sylvestre Bergier, 17181790. Des Monts-Jura à Versailles, le parcours d'un apologiste du $18^{e}$ siècle, Paris, H. Champion, 2010. 
pour commencer à dire du bien de l'Électricité. mais convenez que si je finis par lui devoir, elle n'obligera pas un ingrat, et que pour faire fortune, elle ne pouvoit s'y prendre mieux que de guérir le Confesseur de deux Rois et d'une Reine ${ }^{33}$. » En revanche, comme la plupart des malades nobles, le comte de Boisgelin n'est pas nommé, alors même que le mémoire ne mentionne pas les échanges qu'il a eus avec son médecin Bouvart à propos d'effets indésirables du traitement. Celui-ci le rassurait : "Les érections et l'éjaculation qui est arrivée en dernier lieu, sont sans doute les effets de l'irritation produite par l'électrisation; mais jusqu'ici je les regarde plutôt comme avantageux que nuisibles, à moins qu'ils ne se répétassent plusieurs fois ${ }^{34}$."

Au moment de la publication, l'histoire du malade et de la maladie est reformulée pour respecter la bienséance et la discrétion promise par le médecin mais aussi pour présenter certains échecs thérapeutiques comme le résultat de comportements inappropriés du malade. La mise en valeur de la faiblesse morale ou de l'intempérance du malade peut permettre de relativiser l'échec du nouveau traitement et d'en faire porter la responsabilité au malade. Les excès des plaisirs de l'amour, l'ivrognerie ou la voracité sont les principaux thèmes de ces mises en accusation.

Le dossier de $\mathrm{M}^{\mathrm{me}}$ Boitel, qui réside à Compiègne, contient une correspondance qui permet d'éclairer ces enjeux ${ }^{35}$. Frappée de paralysie, elle s'est présentée au traitement de Mauduyt le 18 février 1778, accompagnée de son médecin ordinaire Lafisse. Quelques jours plus tard, celui-ci fait parvenir à Mauduyt le récit de la maladie qu'il a établi avec la malade. Plusieurs mois après la fin du traitement électrique, lorsque il s'apprête à publier son mémoire, Mauduyt s'enquiert de l'état de la malade. Il reçoit alors une réponse d'une de ses proches. Elle lui fait part d'une interprétation particulière de la maladie : "il faut cependans : monsieur; que je vous avouë (autent part confience que part levive interait que je prend a mde boitel, quelle dine : soupe et se couche tres tarre fais lusage de grand vins et de liqueurs [...] : un empire ne laferait pas convenir de

33. BnF, Nouvelles acquisitions françaises, ms. 10852, "Correspondance de l'abbé Maudoux ", fol. 90.

34. SRM 118, dossier 61.

35. SRM 118, dossier 6. 
ces tores : mais il nansont pas moins raielle [...] que la confidance que je vous fais sur le genre de vie de mde boitel : soit dit entre nous je vous empris; monsieur; peut-etre ne déaprouverait-elle pas; peut-etre aussi le trouveroit elle mauvais. » Mauduyt peut-il publier cette confidence, qui exonère l'électricité de l'échec du traitement, sans trahir la confiance de la confidente et de la malade? Dans le mémoire imprimé, le cas n'est pas anonyme. Mauduyt indique le lieu de résidence de la malade et le nom de son médecin. 11 y fait également référence à la lettre de son amie : « la personne qui écrivait ajoutait qu'à la vérité $\mathrm{M}^{\mathrm{me}}$ Boitel n'avait pas observé le régime que je lui avais recommandé, qu'elle assistait fréquemment à des repas et prolongeait souvent les veilles fort avant dans la nuit. Cette lettre est annexée au journal " (p. 230). Seule la consommation excessive d'alcool n'est pas évoquée.

Mauduyt est plus discret dans le cas de Madame Richer, veuve Caron, qui souffre de surdité. C'est dans une lettre de la malade que son intimité est dévoilée : "vous savez Monsieur que je vous et deja prevenus que je sui d'un temps peramant d'une forse a lextreme; je vous direr que soit que lectriciter qui est propre a fortifier les temps peramant foible devienne prejudiciable au mien... que de puis que je vas chez vous; les chaleurs que jai pour l'ordinaire dans les nuits qui exite des agitation dans la matrise on eter beaucoup plus frequent et jusquat deux fois dans les meme nuit. " La malade insiste sur la honte qu'il y a à raconter et sur le nécessaire secret qu'elle attend de la part du médecin et de la confiance qu'elle lui fait : " [...] je ne vous parle de tout ce cy; pour que vous ayez la bonter de bien examiner ce qui ceroit le plus apropos que je fas; je pense en vous parlant Monsieur, que je parle à une personne respectable et qui mérite la confiance, je vous prie que si vous vous consultez avec quell qun; de ne point me faire connoitre, car je ne sauroit vous exprimer la honte que jai de me voire telle que je sui, et le chagraint et meprit que j'ai éprouver pour ce sujet par la personne que je vous ai nomer sidesû; ce qui m'avoit fais promoit de plutot mourire que de ne jamais en parler a auqun medecin...» Mauduyt a pris le soin de plier et de cacheter le coin de la lettre pour en masquer la signature. Trois mois plus tard, dans le journal du traitement, il écrit : " je ne vois rien quand je lui parle, cette femme se trouble, je ne sai pourquoi et ne m'entend pas, je lui ai 
fait parler par d'autres personnes et elle a entendu assez bien. » Et quelques jours après : " elle entend assez ce matin pour suivre une conversation avec quelqu'un assis a coté d'elle qui lui parle fort haut et de tout prêt. mais aussitôt que je veux lui parler, sa frêle cervelle se démonte, elle rougit et ne m'entend pas où beaucoup moins que tout autre. " Dans le mémoire imprimé, le nom de la malade est mentionné. En revanche, la lettre de la malade et les effets utérins de l'électrisation ne sont pas évoqués, ni même la gêne ou la timidité de la malade. On y trouve en revanche un élément qui ne figure pas dans le dossier manuscrit qui pourrait être une transposition religieuse des transports sexuels. Mauduyt insiste sur le fait que la malade est " habituée à fréquenter les lieux saints » et à " assister aux sermons » (p. 385) ${ }^{36}$.

Le " mémoire sur le traitement électrique administré à quatre-vingt-deux malades » résulte d'un dispositif complexe d'expérimentation thérapeutique. Celle-ci ne peut être réduite à un processus impersonnel et aveugle d'établissement du pouvoir médical et d'un savoir administratif. La nécessité d'entendre, de raconter et de lire la maladie dépasse le cadre de la mise en forme tabulaire qui doit synthétiser l'expérience. La polyphonie des récits et la multiplicité des supports qui apparaissent dans le mémoire traduisent aussi l'autonomie des malades dans l'interprétation de leurs maux et dans leurs recours thérapeutiques. La publication des journaux et des récits de maladies qu'ils contiennent nécessite de la part du médecin qui les met en forme des négociations et des arbitrages où s'exprime son statut incertain. La diversité des sources qui nous renseignent sur le processus d'expérimentation de l'électricité révèle les tensions qui traversent le monde médical de la fin du $18^{\mathrm{e}}$ siècle tant dans ses conceptions que dans ses pratiques.

François ZANETTI

36. Alexandre Wenger, "From medical case to narrative fiction : Diderot's $L a$ Religieuse ", dans Medicine and narration in the $18^{\text {th }}$ century, dir. Sophie Vasset, Oxford, SVEC, 2013, p. 17-30. 
TABLEAU DES MALADES QUI ONT ETE ELECTRISES. $\begin{array}{llllllllllllllllll}P & A & R & A & G & R & A & P & H & E & P & R & E & M & I & E & R\end{array}$ $P A R A$ L $\quad S \quad I E S$
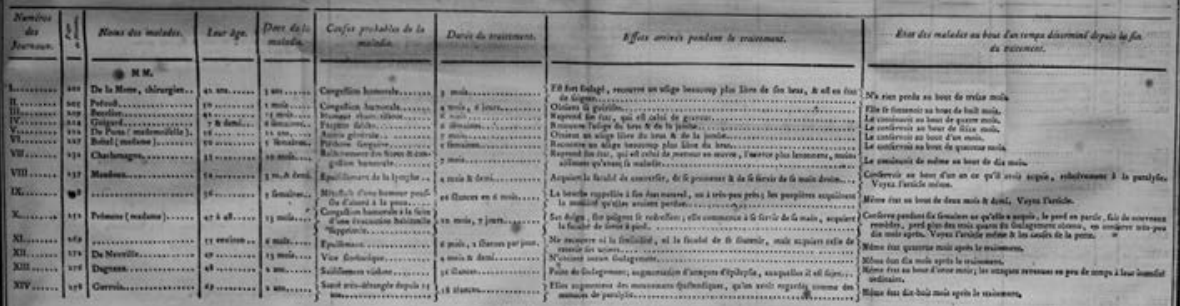

$$
\begin{array}{llllllllllllllllllllll}
P & A & R & A & G & R & A & P & H & E & S & E & C & O & N & D .
\end{array}
$$

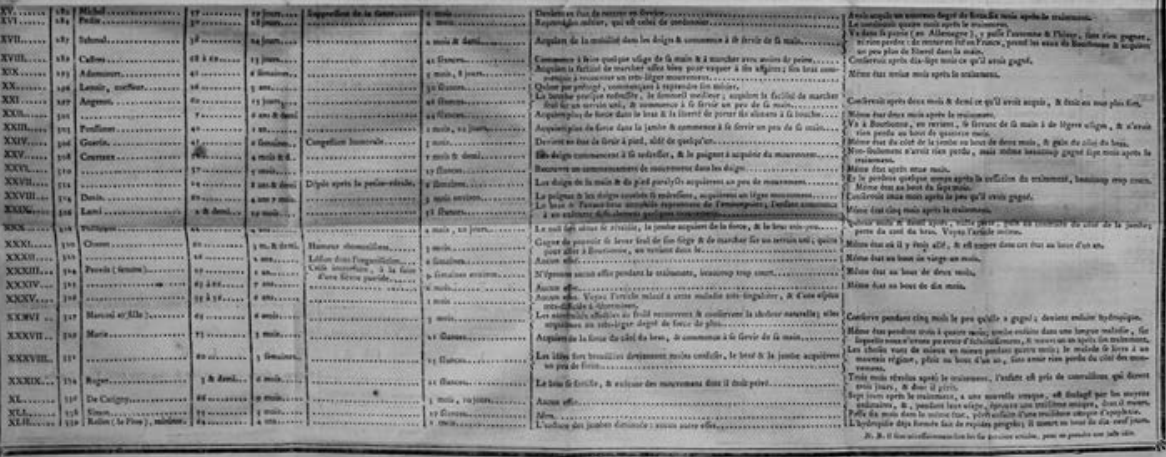

(C) Bibliothèque de l'académie nationale de médecine

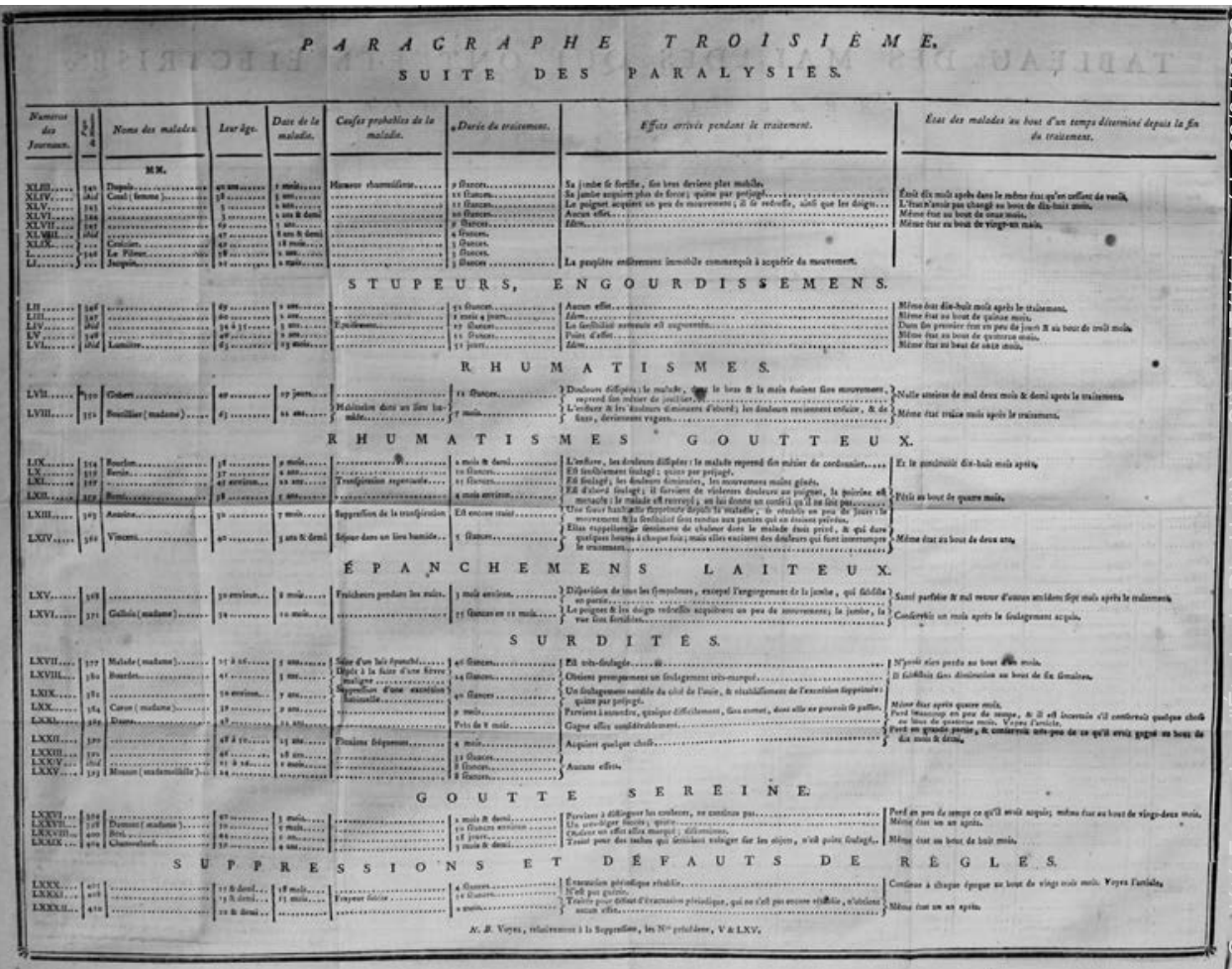

